\title{
Long-term survival of a breast cancer patient with extensive liver metastases upon immune and virotherapy: a case report
}

\begin{abstract}
Liver metastases in breast cancer are associated with a poor prognosis. We report longterm survival of a patient with breast cancer and liver metastases. After operation the patient declined further standard therapy. Instead, she was treated with local hyperthermia, Newcastle disease virus and dendritic cell vaccination at the Immunological and Oncological Center Cologne (IOZK), Germany. A continuous high quality of life was reported and the patient survived more than 66 months after initial diagnosis. No recurrence or further metastases developed under treatment. Following treatment, a long-lasting tumor-reactive memory T-cell responsiveness could be documented. This possibly explains the favorable course of disease. Since this combination of therapies is not restricted to a particular tumor type, further exploration is warranted.
\end{abstract}

Keywords: breast cancer • dendritic cell vaccination • hyperthermia • immunotherapy

- Newcastle disease virus

In Germany, breast cancer is by far the most frequent malignancy in women with an incidence of 70,000 per year [1]. Breast cancer with liver metastases is currently considered incurable and has a poor prognosis. Median survival is 6 months without treatment and 14-22 months with chemotherapy [2]. Current guidelines [3] provide a survey of conventional treatment, including surgery, hormone-, chemo- and radio-therapy as well as antibodies. Unfortunately, conventional treatment has severe side effects that can impair quality of life. By contrast, specific immunotherapy is devoid of severe side effects since it affects only malignant cells and spares the healthy tissue.

Dendritic cell (DC) mediated anti-tumor vaccination is an important immunotherapeutic strategy. It was shown to induce specific T-cell-mediated anti-tumor responses in various malignancies including breast cancer. Curigliano et al. [4] consider DC vaccination for breast cancer 'a clinical reality'. Gelao et al. [5] state that immunotherapy is "now widely recognized as a key element in the treatment of cancer, including breast cancer."
They provide a comprehensive survey of preclinical studies and more than 20 clinical trials on DC vaccination in breast cancer.

Another promising approach is the use of oncolytic viruses that preferentially replicate in tumor cells. For example, oncolytic Newcastle disease virus (NDV) is an avian RNA paramyxovirus with a high safety profile in cancer patients. It's tumor-selective replication, anti-tumor cytotoxicity and immunostimulatory properties make NDV particularly suited for fighting human cancer [6].

Hyperthermia has been used for the treatment of a diverse range of solid tumors. It affects the immune system [7] by activating natural killer cells as well as DCs. Radiofrequency hyperthermia also provokes the expression of heat-shock proteins and may lead to apoptosis in malignant cells [8].

This study provides an account of a combined treatment with hyperthermia, oncolytic virotherapy and DC vaccination. Recently, we reported a case of long-term remission of prostate cancer with extensive bone metastases, using a similar treatment approach [9].
Volker Schirrmacher ${ }^{1}$, Wilfried Stücker', Maria Lulei', Akos-Sigmund Bihari' \& Tobias Sprenger ${ }^{* 1}$ 'Immunological \& Oncological Center, 30-32 Hohenstaufenring, Cologne D-50674, Germany *Author for correspondence: sprenger@iozk.de 


\section{Case report}

\section{Case history}

In October 2009, a 70-year-old patient presented at the Immunological and Oncological Center (IOZK; Cologne, Germany) with invasive ductal breast cancer and primary liver metastases. The patient had previously undergone segmental resection of the left breast and extirpation of axillary lymph nodes in October 2009, shortly after initial diagnosis. The postsurgical staging was $\mathrm{pT} 1 \mathrm{c} \mathrm{pN} 0(0 / 10) \mathrm{M} 1 \mathrm{~L} 0 \mathrm{~V} 0$ R0 G2. The hormone receptor status was ER $80 \%$, PR 5\%, HER $2 /$ Neu 50\% (Score 3). No metastases were found in chest $\mathrm{x}$-ray and bone scintigraphy. Ultrasound revealed at least six liver metastases that were confirmed with biopsy. Histologically, the metastases could be attributed without doubt to the breast cancer. According to German guidelines, the patient was advised to take letrozole and possibly chemotherapy in case of progress of liver metastases under treatment. The patient, however, refused conventional treatment altogether and opted for a specific immunotherapy.

\section{Immunotherapy}

Between December 2009 and April 2010, the patient was treated at the IOZK with radiofrequency hyperthermia (RHT) of the liver combined with systemic oncolytic NDV virotherapy approximately twice a month. In addition, the patient received five vaccinations with DCs that were pulsed with lysate from NDV-infected breast cancer cells (oncolysate). Between October 2010 and December 2012, the patient was treated with RHT of the liver and virotherapy approximately once a month to sustain the immune response. In May 2012, ${ }^{18}$ F-FDG PET revealed progression of the metastases in size and metabolism. Also an Enzyme-Linked ImmunoSpot Assay (ELISPOT) revealed a decrease in the number of cancer-reactive memory $\mathrm{T}$ cells. For these reasons, vaccination therapy was resumed. The patient received another three vaccinations with NDV-activated DC1 polarized cells [10] in May 2012, December 2012 and February 2013. Between November 2013 and June 2014, the patient received monthly treatments with RHT and NDV. In April and May 2014 and from October 2014 onward, treatment was administered every four to six weeks with RHT, NDV and moderate whole-body hyperthermia.

Hyperthermia was administered with the Oncothermia EHY-2000 device (Oncotherm GmbH, Troisdorf, Germany) with a radiofrequency of $13 \mathrm{MHz}$. In total, the patient received 52 hyperthermia treatments to the liver. The duration of the sessions was $50 \mathrm{~min}$ each, starting at $50 \mathrm{~W}$ and increasing to maximum
$80 \mathrm{~W}$ per session, the patient received $10^{7}$ plaqueforming units of NDV (oncolytic strain MTH-68) intravenously to target the heated tumor tissue.

DCs were differentiated from autologous blood monocytes with granulocyte-macrophage colonystimulating factor and IL-4. For the first five vaccinations, immature DCs were pulsed with a lysate from the human breast cancer cell line MDA-MB- 453. The lysate contained equal parts of previously NDVinfected cells and cells that were pretreated by hyperthermia. Subsequent to DC maturation, the oncolysate-pulsed cells were administered intradermally. For the last three vaccinations, immature DCs were activated with NDV to provide DC1 polarized cells. Moderate whole-body hyperthermia increased the body temperature to $38.5-40.0^{\circ} \mathrm{C}$ (Infrared device, Heckel-HT2000; Heckel Medizintechnik GmbH, Esslingen, Germany).

\section{Outcome \& follow-up}

In November 2010, ${ }^{18} \mathrm{~F}$-FDG PET revealed seven metastases in the liver. Another ${ }^{18} \mathrm{~F}-\mathrm{FDG}$ PET examination in May 2012 showed progression of these metastases in size and metabolism but revealed no further distant metastases. In July 2014 an ${ }^{18} \mathrm{~F}-\mathrm{FDG}$ positron-emission-tomography/computed tomography (PET/CT) evidenced no relevant progression in comparison to the ${ }^{18} \mathrm{~F}-\mathrm{FDG}$ PET from May 2012. Also, local recurrence and further distant metastases were ruled out.

To determine the success of the vaccinations and to evaluate the need for further vaccinations, the patient's T-cell responses were monitored. The test was performed with isolated $\mathrm{T}$ cells and oncolysatepulsed DCs from the peripheral blood in a short-term assay allowing to quantify an immunological antitumor memory T-cell response. The read-out was an Enzyme-Linked IFN- $\gamma$ ImmunoSpot (ELISPOT) assay (Autoimmun Diagnostika GmbH, Strassberg, Germany). The T cells secreting IFN- $\gamma$ upon shortterm $(40 \mathrm{~h})$ contact with oncolysate-pulsed autologous DCs were quantified. The results are illustrated in Figure 1. They begin with the first test prior to vaccination and end with the last test performed in January 2014. Memory T-cell responses increased following vaccinations but decreased in 2012. After resumption of DC vaccinations, a profound memory T-cell response could be reconstituted. These data coincide with the clinical course: a poor T-cell response in May 2012 corresponded with progress of liver metastases seen in the PET. After re-establishment of a strong memory T-cell response, the liver metastases remained stable as revealed by PET/CT in June 2014. 


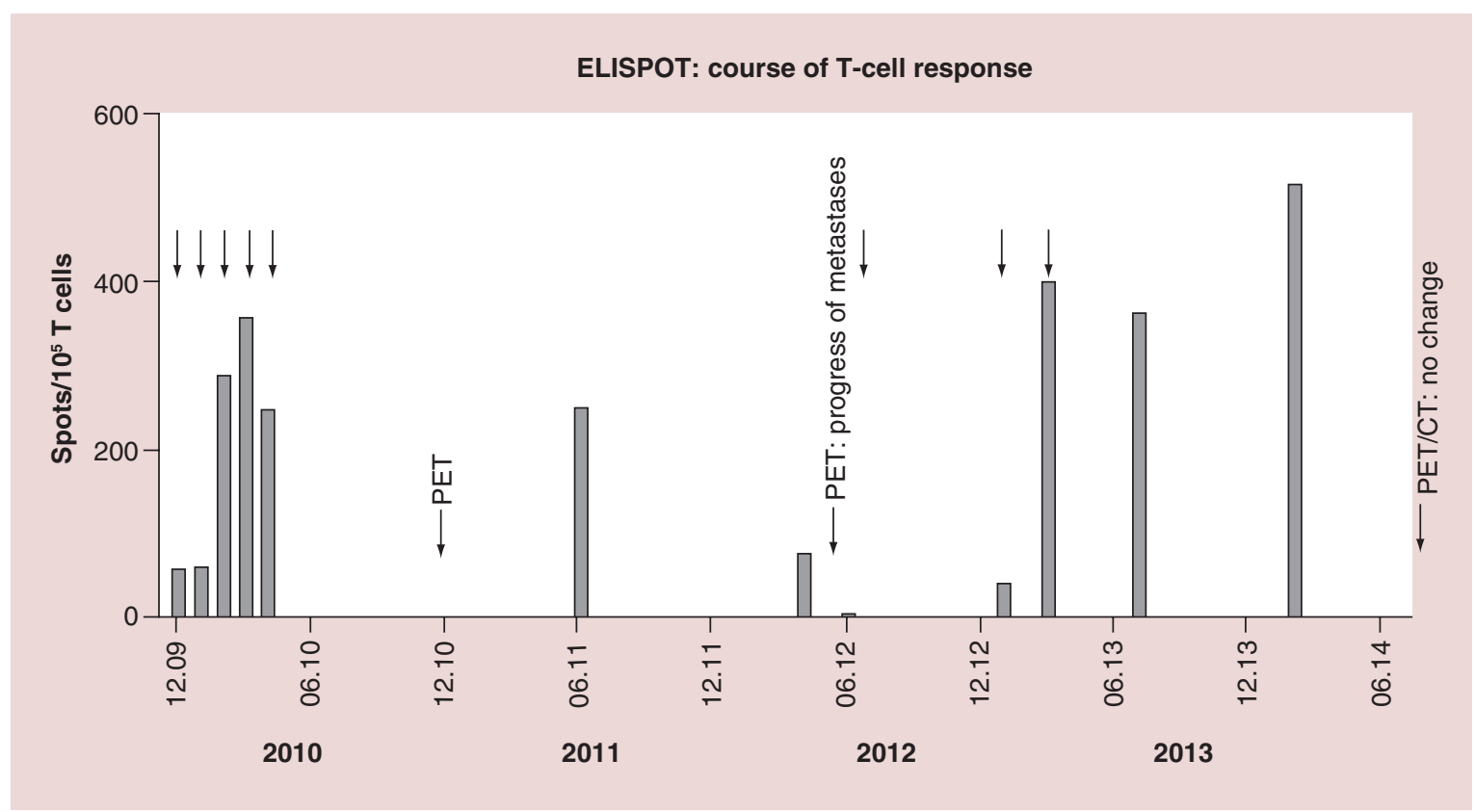

Figure 1. Course of T-cell response in Enzyme-Linked ImmunoSpot Assay. $10^{5}$ autologous T cells activated via autologous mature DC pulsed with lysate from Newcastle disease virus-infected breast cancer cells in a short time IFN- $\gamma$ ELISPOT assay. The number of IFN- $\gamma$-producing activated memory $T$ cells is shown, expressed as the difference between background ( $T+D C$ without oncolysate) and test sample ( $T+D C$ with oncolysate). Arrows at the top represent DC vaccination, arrows at the bottom represent medical imaging.

CT: Computed tomography; DC: Dendritic cell; ELISPOT: Enzyme-Linked ImmunoSpot Assay.

The treatment was well tolerated. A continuous high quality of life was reported. The patient runs her household by herself. She was never hospitalized after initial operation. By now, she has survived more than 66 months after initial diagnosis.

Written informed consent was obtained from the patient for the publication of this case report.

\section{Discussion}

Considering an expected median survival of 6 months in patients with breast cancer and distant metastases in the liver, the long-term (>66 months) survival as well as the high quality of life maintained in this patient is very surprising. Since the patient did not receive any conventional therapy of the liver metastases and did not undergo any relevant changes of lifestyle, the effect most likely has to be attributed to this specific immunotherapy.

The concept of immunological surveillance of cancer has been proposed for the first time more than four decades ago by Burnet [11]. In 2004, the prevalent concept of immunoediting [12] was proposed which describes the complex interactions over time between a tumor and the host's immune system.

A CD8 ${ }^{+}$cytotoxic T-cell response seems to be crucial to control tumor growth. The presence of tumor-infiltrating $\mathrm{CD}^{+} \mathrm{T}$ cells predicted a better clinical outcome in breast cancer [13]. A cross-talk between adaptive and innate immune cells was apparently capable to influence metastatic spread in breast cancer [14]. In addition, an establishment of anti-tumor T-cell memory has been emphasized to be of importance for long-term survival [15]. Cancerreactive memory $\mathrm{T}$ cells accumulate especially in the bone marrow of breast cancer patients [16]. The repertoire of specificities of tumor-reactive memory $\mathrm{T}$ cells in the bone marrow from breast cancer patients is polyvalent and highly individual [17]. Such cells can be reactivated ex vivo by autologous DCs pulsed with autologous tumor lysate and show functional reactivity, including autologous human tumor xenotransplant rejection in NOD/SCID mice [18]. Such cells can also be reactivated in situ, for instance, by postoperative vaccination with NDV-modified autologous tumor vaccine (ATV-NDV). Tumor cell number and viability were reported to be of importance as quality and efficacy parameters in patients with breast cancer [19].

In this case, a sustained immune T-cell-mediated anti-tumor memory response was detected and documented over time. This was probably induced by the combination of RHT, oncolytic virotherapy with NDV and specific DC vaccination. Naturally, it is difficult to conclude how and to what extent the respective measures contributed to the establishment of the anti-tumor memory. The 
combination of different immunologic strategies appears reasonable to obtain synergistic effects and to combat tumor-induced immune escape mechanisms including T-cell anergy.
RHT hyperthermia and NDV were combined because RHT has been reported to enhance virus tumor targeting [20] and replication [21]. Also, vaccination was combined with moderate whole body

Executive summary

- Breast cancer is the most frequent malignancy in women.

- Liver metastases deteriorate the prognosis.

- Conventional treatments like surgery, hormone-, chemo- and radio-therapy or antibodies often have severe side effects.

- Specific immunotherapy only affects malignant cells and therefore has no severe side effects.

- Dendritic cell (DC) vaccination, the use of oncolytic viruses and hyperthermia are important components of the immunotherapeutic strategy applied.

Case report

- A 70-year-old patient suffering from invasive ductal breast cancer with primary liver metastases underwent resection of the breast.

- Postoperative staging was pT1c pN0 (0/10) M1 L0 V0 R0 G2, hormone receptor status was estrogen receptor $80 \%$, progesteron receptor $5 \%, \mathrm{HER} 2 / \mathrm{Neu} 50 \%$ (Score 3 ).

- The patient refused systemic treatment of the liver metastases against medical advice and opted for immunotherapy.

Immunotherapy

- Between December 2009 and April 2010, the patient was treated with radiofrequency hyperthermia (RHT) of the liver combined with systemic oncolytic Newcastle disease virus (NDV) virotherapy and received five vaccinations with oncolysate-pulsed DCs.

- Subsequently, monthly sessions of RHT and NDV were realized to sustain the immune response.

- The patient received another three vaccinations between May 2012 and February 2013.

- Between November 2013 and June 2014, the patient received monthly treatments with RHT and NDV.

- In April and May 2014 and from October 2014 onward, treatments were performed every 4 to 6 weeks with RHT, NDV and moderate whole-body hyperthermia.

Outcome \& follow-up

- The T-cell response toward the vaccine (oncolysate-pulsed DCs) was monitored via Enzyme-Linked Immunospot Assay.

- After the first five vaccinations, a good memory T-cell response could be documented.

- Controls in 2012 revealed a decrease in the number of reactive T cells, coinciding with progress of the liver metastases.

- After further three vaccinations, the memory T-cell response was successfully restored.

- The last control in January 2014 revealed a long-lasting memory T-cell response.

- A PET/computed tomography showed no further progress of the liver metastases in June 2014. Local recurrence and further distant metastases were ruled out.

- The treatment was well tolerated. A continuous high quality of life was reported. The patient runs her household by herself. She was never hospitalized after initial operation. By now, she has survived more than 66 months after initial diagnosis.

\section{Discussion}

- The long-term survival as well as the high quality of life maintained in this patient most likely has to be attributed to immunotherapy because she did not receive any conventional systemic treatment for the liver metastases.

- The importance of the immune system during the development of cancer is reflected in the prevalent concept of immunoediting.

- The patient developed a cancer-reactive memory T-cell repertoire. This could be followed over the years along with the treatment. There was no evidence for T-cell anergy or immune escape.

- The different components of the immunotherapy were combined in such a way as to obtain possible synergistic effects.

\section{Conclusion}

- Immunotherapy combining RHT, NDV and DC vaccination led to a long-lasting cancer-reactive memory T-cell repertoire and to a favorable course with stable disease in this particular patient.

- The combination of RHT, NDV and DC vaccination appears as promising tools for future treatments.

- The identification of predictive factors for patient's responsiveness will be important. 
hyperthermia to enhance DC function [22]. Viral infection of tumor cells and hyperthermia cause an endoplasmic reticulum stress response, modify the surface properties of tumor cells and induce immunogenic tumor cell death mechanisms [23].

Host DCs process the viral oncolysate and crosspresent autologous tumor antigens thereby priming specific $\mathrm{T}$ cells. In addition, pre-existing tumor-reactive memory $\mathrm{T}$ cells are likely to become re-activated and expanded. DCs pulsed with viral oncolysate were shown to potently stimulate autologous $\mathrm{T}$ cells from breast cancer patients [24]. Both the quantity and the quality of the memory T-cell response was augmented in comparison to stimulation by DCs pulsed with tumor lysate without NDV. T cells responding to viral oncolysate produced not only more IFN- $\gamma$ but also more IFN- $\alpha$ and more IL-15 [24]. Ideally, the tumor-reactive memory T-cell repertoire comprises central memory $\mathrm{T}$ cells and effector memory $\mathrm{T}$ cells $[25]$ and include $\mathrm{CD}^{+}$ helper $\mathrm{T}$ cells and $\mathrm{CD} 8^{+}$cytotoxic $\mathrm{T}$ cells.

Our concept combines preconditioning of the patient's immune system by RHT and NDV with specific anti-tumoral DC vaccination. This appears as a viable strategy based on involvement of oncolysate-specific helper $\mathrm{T}$ cells causing upregulation at the vaccination site of the chemokine CCL3 thereby improving DC migration [25].

\section{Conclusion}

Taken together, the combination of different immunotherapeutic strategies provoked a long-lasting anti-tumor memory $\mathrm{T}$-cell response and led to a favorable course in this particular patient. To date, hundreds of patients with various malignancies were treated at IOZK applying a similar strategy. This has led to the establishment of positive ELISPOT reactivity in about $80 \%$ of the vaccinated patients. According

\section{References}

Papers of special note have been highlighted as:

- of interest; $\bullet \bullet$ of considerable interest

1 Robert Koch-Institut (Hrsg) und die Gesellschaft der epidemiologischen Krebsregister in Deutschland e.V. (Hrsg). Krebs in Deutschland 2009/2010 (9th Edition). Robert Koch Institute, Berlin, Germany (2013).

2 Atalay G, Biganzoli L, Renard F et al. EORTC Breast Cancer and Early Clinical Studies Groups. Clinical outcome of breast cancer patients with liver metastases alone in the anthracycline-taxane era: a retrospective analysis of two prospective, randomised metastatic breast cancer trials. Eur. J. Cancer 39(17), 2439-2449 (2003).

3 Interdisziplinäre S3-Leitlinie für die Diagnostik, Therapie und Nachsorge des Mammakarzinoms. www.awmf.org to our experience, such ELISPOT memory T-cell reactivity correlates with a positive clinical outcome, even in patients with advanced stage cancer. Since we treat single patients with unequal preconditions, it is impossible to quantify success rates.

Considering the frequent clinical response and the low level of side effects, this approach deserves further investigation. In future, the identification of predictive factors for patient's responsiveness would help to increase the response rate. Single case studies, such as the present one, can provide innovation and increase of medical knowledge. We are convinced that single case use, as approved by the German Pharmaceuticals Act, is important for patients and for medical progress.

\section{Financial \& competing interests disclosure}

The authors have no relevant affiliations or financial involvement with any organization or entity with a financial interest in or financial conflict with the subject matter or materials discussed in the manuscript. This includes employment, consultancies, honoraria, stock ownership or options, expert testimony, grants or patents received or pending, or royalties.

No writing assistance was utilized in the production of this manuscript.

\section{Informed consent disclosure}

The authors state that they have obtained verbal and written informed consent from the patient for the inclusion of their medical and treatment history within this case report.

\section{Open access}

This work is licensed under the Creative Commons Attribution-NonCommercial 3.0 Unported License. To view a copy of this license, visit http://creativecommons.org/licenses/bync-nd/3.0/

4 Curigliano G, Criscitiello C, Esposito A et al. Developing an effective breast cancer vaccine: challenges to achieving sterile immunity versus resetting equilibrium. Breast 22(Suppl. 2), S96-S99 (2013).

-• Offers an up-to-date survey of all issues concerning immunotherapy in breast cancer.

5 Gelao L, Criscitiello C, Esposito A et al. Dendritic cellbased vaccines: clinical applications in breast cancer. Immunotherapy 6(3), 349-360 (2014).

-. This is the latest and most comprehensive review of clinical application of dendritic cell (DC) vaccination in breast cancer.

6 Fournier P, Schirrmacher V. Oncolytic Newcastle disease virus as cutting edge between tumor and host. Biology (Basel) 2(3), 936-975 (2013). 
-. Reviews 50 years of research on Newcastle disease virus, focusing on its tumor-selective replication, its anti-tumor cytotoxicity and its immunostimulatory properties.

7 Frey B, Weiss EM, Rubner Y et al. Old and new facts about hyperthermia-induced modulations of the immune system. Int. J. Hyperthermia 28(6), 528-542 (2012).

8 Hildebrandt B, Wust $\mathrm{P}$, Ahlers $\mathrm{O}$ et al. The cellular and molecular basis of hyperthermia. Crit. Rev. Oncol. Hematol. 43, 33-56 (2002).

9 Schirrmacher V, Bihari AS, Stücker W, Sprenger T. Longterm remission of prostate cancer with extensive bone metastases upon immuno- and virotherapy: a case report. Oncol. Lett. 8(6), 2403-2406 (2014).

10 Zaslavsky E, Hershberg U, Seto J et al. Antiviral response dictated by choreographed cascade of transcription factors. J. Immunol. 184, 2908-2917 (2010).

- This study from systems biology describes how infection of human DCs by Newcastle disease virus cause re-programming of the DCs toward a DC1 phenotype. This uninhibited antiviral cellular response involves 24 transcription factors causing upregulation of 779 genes.

11 Burnet FM. The concept of immunological surveillance. Prog. Exp. Tumor Res. 13, 1-27 (1970).

12 Dunn GP, Old LJ, Schreiber RD. The three Es of cancer immunoediting. Annu. Rev. Immunol. 22, 329-360 (2004).

13 Mahmoud SM, Paish EC, Powe DG et al. Tumor-infiltrating $\mathrm{CD}^{8+}$ lymphocytes predict clinical outcome in breast cancer. J. Clin. Oncol. 29(15), 1949-1955 (2011).

14 DeNardo DG, Coussens LM. Inflammation and breast cancer. Balancing immune response: crosstalk between adaptive and innate immune cells during breast cancer progression. Breast Cancer Res. 9(4), 212 (2007).

15 Schirrmacher V, Feuerer M, Beckhove P, Ahlert T, Umansky $\mathrm{V}$. T cell memory, anergy and immunotherapy in breast cancer. J. Mammary Gland Biol. Neoplasia 7, 201-208 (2002).

16 Feuerer M, Rocha M, Bai L et al. Enrichment of memory $\mathrm{T}$ cells and other profound immunological changes in the bone marrow from untreated breast cancer patients. Int. J. Cancer 92, 96-105 (2001).

17 Sommerfeld N, Schütz F, Sohn C et al. The shaping of a polyvalent and highly individual T-cell repertoire in the bone marrow of breast cancer patients. Cancer Res. 66, 8258-8265 (2006).

- Describes the cancer-reactive memory T-cell repertoire of breast cancer patients as being polyvalent and highly individual. To reactivate this would require an autologous approach.

18 Feuerer M, Beckhove P, Bai L et al. Therapy of human tumors in NOD/SCID mice with patient derived reactivated memory T cells from bone marrow. Nat. Med. 7 , 452-458 (2001).

-. Describes the re-activation in vitro of cancer-reactive memory $T$ cells from breast cancer patients with autologous tumor lysate pulsed DCs and their therapeutic capacity following transfer into immune incompetent mice bearing autologous tumor transplants. It underlines the relevance of cancer-reactive memory $\mathrm{T}$ cells and their mode of function in vivo.

19 Ahlert T, Sauerbrei W, Bastert G et al. Tumor cell number and viability as quality and efficacy parameters of autologous virus-modified cancer vaccines. J. Clin. Oncol. 15, 1354-1366 (1997).

- This early clinical study of postoperative vaccination of breast cancer patients with autologous virus-modified cancer vaccine concludes that tumor cell number and viability are important for clinical efficacy.

20 Chang E, Chalikonda S, Friedl J et al. Targeting vaccinia to solid tumors with local hyperthermia. Hum. Gene Ther. 16, 435-444 (2005).

21 Eisenberg DP, Carpenter SG, Adusumilli PS et al. Hyperthermia potentiates oncolytic herpes viral killing of pancreatic cancer through a heat shock protein pathway. Surgery 148, 325-334 (2010).

22 Knippertz I, Stein MF, Dörrie J et al. Mild hyperthermia enhances human monocyte derived dendritic cell functions and offers potential for applications in vaccination strategies. Int. J. Hyperthermia 27, 591-603 (2011).

23 Hou W, Zhang Q, Yan Z et al. Strange attractors: DAMPs and autophagy link tumor cell death and immunity. Cell Death Dis. 4, e966 (2013).

24 Bai L, Koopmann J, Fiola C et al. Dendritic cells pulsed with viral oncolysate potently stimulate autologous $\mathrm{T}$ cells from cancer patients. Int. J. Oncol. 21(4), 685-694 (2002).

25 Mitchell DA, Batich KA, Gunn MD et al. Tetanus toxoid and CCL3 improve dendritic cell vaccines in mice and glioblastoma patients. Nature 519 (7543), 366-369 (2015).

- $\quad$ Suggests that $T$ helper cells, upon recruitment to the site of DC vaccination, can improve DC migration through upregulation of the chemokine CCL3. 